\title{
溶剂-导体界面电子转移溶剂重组能的球-界面模型
}

\author{
涂吉研李象远* 傅克祥 何福城 \\ (四川大学化工学院, 成都 610065)
}

\begin{abstract}
摘要: 在连续介质理论基础上, 根据热力学基本原理, 用一个外加电场 $\boldsymbol{E}_{\mathrm{ex}}$ 将非平衡态 $2\left[\boldsymbol{E}_{2}^{\mathrm{non}}, \boldsymbol{D}_{2}^{\mathrm{non}}\right]$ 变成约束平 衡态 $\left[\boldsymbol{E}_{2}^{*}, \boldsymbol{D}_{2}^{*}\right]$, 推导出了正确普适的溶剂重组能公式. 基于球-界面近似, 推导出了正确的溶剂-导体界面电子 转移溶剂重组能公式. 和 Marcus 的公式相比, 本文的结果多了 $\left(\varepsilon_{\mathrm{s}}-\varepsilon_{\mathrm{op}}\right) /\left(\varepsilon_{\mathrm{op}}\left(\varepsilon_{\mathrm{s}}-1\right)\right)$ 因子. 对极性溶剂, 预测的溶剂 重组能约为 Marcus 模型所得结果的一半. 以 $\mathrm{C} 343$ (Coumarin 343)- $\mathrm{TiO}_{2}$ 体系为算例, 计算了溶剂重组能并与实 验值进行了比较.
\end{abstract}

关键词: 非平衡溶剂化; 约束平衡; 溶剂重组能; 界面 中图分类号：0641

\section{A Sphere-Interface Model for the Solvent Reorganization Energy in an Electron Transfer Reaction at the Solvent/Conductor Interface}

\author{
TU Zhe-Yan LI Xiang-Yuan* FU Ke-Xiang HE Fu-Cheng \\ (College of Chemical Engineering, Sichuan University, Chengdu 610065, P. R. China)
}

\begin{abstract}
Based on the continuum medium theory and the basic principles of thermodynamics we used an extra electric field $\boldsymbol{E}_{\text {ex }}$ to change the non-equilibrium salvation state $2\left[\boldsymbol{E}_{2}^{\text {non }}, \boldsymbol{D}_{2}^{\text {non }}\right]$ to a constrained equilibrium state $\left[\boldsymbol{E}_{2}^{*}, \boldsymbol{D}_{2}^{*}\right]$. A novel expression of solvent reorganization energy was deduced. Furthermore, by adopting the sphere-interface approximation we deduced the formula for the solvent reorganization energy of the electron transfer reaction occurring at the interface of the conductor and the solvent. Compared to the traditional Marcus model our result was scaled by a factor of $\left(\varepsilon_{\mathrm{s}}-\varepsilon_{\mathrm{op}}\right) /\left(\varepsilon_{\mathrm{op}}\left(\varepsilon_{\mathrm{s}}-1\right)\right)$ which is about 0.5 in a polar solvent. C343(Coumarin 343$)-\mathrm{TiO}_{2}$ was used as an example and the analytical results using our model were discussed with respect to experimental measurements.
\end{abstract}

Key Words : Non-equilibrium solvation; Constrained equilibrium; Solvent reorganization energy; Interface

迄今为止, 连续介质模型依然是理论计算溶剂 效应的主要工具. 溶液中的电子转移、光吸收和发射 等众多物理化学过程都与非平衡溶剂化密切相关 ${ }^{[1]}$. 非平衡溶剂化的概念最早由 Marcus 提出 ${ }^{[2,3]}$, 他将溶 剂分子的极化划分为取向极化和电子极化. 在连续 介质模型条件下, 基于对溶剂分子极化的区分, Marcus 采用热力学可逆功的方法推导了非平衡态 自由能 ${ }^{[2-4]}$, 近几十年来被认为是研究电子转移反应 的基础.
为了验证 Marcus 的理论, 上个世纪 80 年代, Closs 和Miller等人 ${ }^{[5-8]}$ 开展了经典的实验测量. 可是, Johnson和 Miller 等人 ${ }^{[7]}$ 在用 Marcus 双球模型估算 联苯-桥体-菜的电子转移溶剂效应时, 发现根据溶 剂重组能实验拟合值反推得到的联苯和菜的平均半 径值比实验数据大很多. 实际上已有很多工作提出 了对 Marcus 理论的疑惑 ${ }^{[7-15]}$. 一些人把理论计算值 与实验值的偏差归处于连续介质模型的缺陷, 可我 们认为这不是问题的关键所在. 近几年来, 我们利用

Received: July 17, 2008; Revised: September 16, 2008; Published on Web: November 3, 2008.

*Corresponding author. Email: xyli@scu.edu.cn; Tel: +8628-85405233.

国家自然科学基金(20533070,20625331)资助项目

C Editorial office of Acta Physico-Chimica Sinica 
Jackson方程 ${ }^{[16]}$, 提出了不同于Marcus的非平衡态自 由能公式 ${ }^{[17-20]}$. 但发现, Jackson方程只适用于介电 性质变化的平衡态之间自由能差计算, 用于非平衡 态自由能计算时仍然存在不足.

本文根据连续介质理论和热力学基本原理推导 出了正确普适的非平衡溶剂化能和溶剂重组能的公 式. 进而应用镜象电荷法推导出了正确的溶剂-导体 界面电子转移溶剂重组能公式. 以 $\mathrm{C} 343-\mathrm{TiO}_{2}$ 为算 例, 溶剂重组能计算结果与实验值符合得较好, 而 Marcus 公式的计算结果约比实验值大了 1 倍.

\section{1 理 论}

\section{1 电子转移溶剂重组能}

连续介质模型中, 用电场 $\boldsymbol{E}$ 和电位移矢量 $\boldsymbol{D}$ 描 述溶质溶剂体系的状态. 溶质电荷分布发生变化, 体 系从平衡态 $1\left[\boldsymbol{E}_{1}^{\mathrm{eq}}, \boldsymbol{D}_{1}^{\mathrm{eq}}\right]$ 变为非平衡态 $2\left[\boldsymbol{E}_{2}^{\mathrm{non}}, \boldsymbol{D}_{2}^{\mathrm{non}}\right]$. 在非平衡态 2 , 溶剂分子的电子极化及时响应溶质 电荷变化, 但溶剂取向极化与原来平衡态相同. 然 而, 取向极化最终会弛豫到平衡态 $2\left[\boldsymbol{E}_{2}^{\mathrm{eq}}, \boldsymbol{D}_{2}^{\mathrm{eq}}\right]$, 这两 个态的差异实际上就是溶剂介质取向极化的差异. 我们将非平衡态 $2\left[\boldsymbol{E}_{2}^{\mathrm{non}}, \boldsymbol{D}_{2}^{\mathrm{non}}\right]$ 的总极化 $\boldsymbol{P}_{2}^{\mathrm{non}}$ 和平衡态 $2\left[\boldsymbol{E}_{2}^{\mathrm{eq}}, \boldsymbol{D}_{2}^{\mathrm{eq}}\right]$ 的总极化 $\boldsymbol{P}_{2}^{\mathrm{eq}}$ 的差记为 $\boldsymbol{P}^{\prime}$, 我们称之为残 余极化(residue polarization), 即

$$
\boldsymbol{P}^{\prime}=\boldsymbol{P}_{2}^{\text {non }}-\boldsymbol{P}_{2}^{\text {eq }}
$$

$\left[\boldsymbol{E}_{2}^{\mathrm{non}}, \boldsymbol{D}_{2}^{\mathrm{non}}\right]$ 之所以称为非平衡态是因为 $\boldsymbol{P}_{2}^{\mathrm{non}}$ 与 $\boldsymbol{E}_{2}^{\mathrm{non}}$ 不 存在线性关系, 即

$$
\boldsymbol{P}_{2}^{\text {non }} \neq \chi_{s} \boldsymbol{E}_{2}^{\text {non }}
$$

式(2)中 $\chi_{\mathrm{s}}$ 是溶剂介质的静态极化率, 我们注意到 $\boldsymbol{P}_{2}^{\mathrm{eq}}=$ $\chi_{\S} \boldsymbol{E}_{2}^{\mathrm{eq}}$, 并定义 $\boldsymbol{E}_{\boldsymbol{P}}=\boldsymbol{E}_{2}^{\mathrm{non}}-\boldsymbol{E}_{2}^{\mathrm{eq}}$ 为 $\boldsymbol{P}^{\prime}$ 产生的极化场. 再由 式(1), 得到式(2)等价于

$$
\boldsymbol{P}^{\prime} \neq \chi_{\underline{s}} \boldsymbol{E}_{\boldsymbol{P}^{\prime}}
$$

Leontovich 在《热力学概论》 ${ }^{21]} \$ 29$ 和\$30 中介绍 了通过外加力场将非平衡态变为约束平衡态的方 法, 是计算非平衡态自由能的基本原理. 如果给以合 适的广义力, 一个非平衡态被约束成一个平衡态, 那 么这个非平衡态自由能 $G^{\mathrm{non}}$ 可以写为 ${ }^{[2]]}$

$$
G^{\mathrm{non}}=G^{*}+\sum_{k} \boldsymbol{x}_{k} \boldsymbol{\xi}_{k}
$$

式(4)中, $G^{*}$ 是包括外加力场的约束平衡态的自由能, $\boldsymbol{x}_{k}$ 和 $\boldsymbol{\xi}_{k}$ 分别是广义位移和广义力, 所以 $\sum_{k} \boldsymbol{x}_{k} \boldsymbol{\xi}_{k}$ 是引 人外加力场所做的功.

基于此热力学原理, 我们给非平衡态 $2\left[\boldsymbol{E}_{2}^{\mathrm{non}}, \boldsymbol{D}_{2}^{\mathrm{non}}\right]$
增加一个外加电场 $\boldsymbol{E}_{\mathrm{ex}}$, 使其变成约束平衡态 $\left[\boldsymbol{E}_{2}^{*}, \boldsymbol{D}_{2}^{*}\right]$. $\boldsymbol{E}_{\mathrm{ex}}$ 的加人, 是为了使式(3)变为

$$
\boldsymbol{P}^{\prime}=\chi_{\mathrm{s}}\left(\boldsymbol{E}_{\mathrm{ex}}+\boldsymbol{E}_{\boldsymbol{P}^{\prime}}\right)
$$

式(5)成立, 就说明成功地将非平衡态 $2\left[\boldsymbol{E}_{2}^{\mathrm{non}}, \boldsymbol{D}_{2}^{\mathrm{non}}\right]$ 变 成了约束平衡态 $\left[\boldsymbol{E}_{2}^{*}, \boldsymbol{D}_{2}^{*}\right]$. 那么, $\boldsymbol{E}_{\mathrm{ex}}$ 可表示为

$$
\boldsymbol{E}_{\mathrm{ex}}=\frac{\boldsymbol{P}^{\prime}}{\chi_{\mathrm{s}}}-\boldsymbol{E}_{\boldsymbol{P}}=\frac{\boldsymbol{P}_{2}^{\mathrm{non}}-\boldsymbol{P}_{2}^{\mathrm{eq}}}{\chi_{\mathrm{s}}}-\left(\boldsymbol{E}_{2}^{\mathrm{non}}-\boldsymbol{E}_{2}^{\mathrm{eq}}\right)
$$

式(6)中 $\boldsymbol{P}_{2}^{\text {non }} 、 \boldsymbol{E}_{2}^{\mathrm{non}}$ 又可以写为

$$
\begin{aligned}
& \boldsymbol{P}_{2}^{\text {non }}=\boldsymbol{P}_{1}^{\text {eq }}+\Delta \boldsymbol{P}_{\mathrm{op}} \\
& \boldsymbol{E}_{2}^{\text {non }}=\boldsymbol{E}_{1}^{\text {eq }}+\Delta \boldsymbol{E}_{\text {op }}
\end{aligned}
$$

式(7)和(8)中, $\boldsymbol{P}_{1}^{\mathrm{eq}}$ 是平衡态 1 的总极化, $\Delta \boldsymbol{P}_{\mathrm{op}}$ 是溶剂 介质电子极化的变化, $\Delta \boldsymbol{E}_{\mathrm{op}}$ 是溶质真空场的变化与 溶剂介质电子极化场的变化之和. 把式(7)和(8)代人 式(6)中得

$$
\begin{array}{r}
\boldsymbol{E}_{\mathrm{ex}}=\frac{\boldsymbol{P}^{\prime}}{\chi_{\mathrm{s}}}-\boldsymbol{E}_{\boldsymbol{P}}=\frac{\Delta \boldsymbol{P}_{\mathrm{op}}-\left(\boldsymbol{P}_{2}^{\mathrm{eq}}-\boldsymbol{P}_{1}^{\mathrm{eq}}\right)}{\chi_{\mathrm{s}}}- \\
{\left[\Delta \boldsymbol{E}_{\mathrm{op}}-\left(\boldsymbol{E}_{2}^{\mathrm{eq}}-\boldsymbol{E}_{1}^{\mathrm{eq}}\right)\right]}
\end{array}
$$

记 $\Delta \boldsymbol{P}_{\text {eq }}=\boldsymbol{P}_{2}^{\text {eq }}-\boldsymbol{P}_{1}^{\text {eq }}, \Delta \boldsymbol{E}_{\text {eq }}=\boldsymbol{E}_{2}^{\text {eq }}-\boldsymbol{E}_{1}^{\text {eq }}, \Delta \boldsymbol{P}_{\text {eq }}$ 和 $\Delta \boldsymbol{E}_{\text {eq }}$ 分别为 平衡态 2 与平衡态 1 的总极化的差和总电场的差. 那么式(9)可写为

$$
\boldsymbol{E}_{\mathrm{ex}}=\frac{\boldsymbol{P}^{\prime}}{\chi_{\mathrm{s}}}-\boldsymbol{E}_{\boldsymbol{P}}=\frac{\Delta \boldsymbol{P}_{\mathrm{op}}-\Delta \boldsymbol{P}_{\mathrm{eq}}}{\chi_{\mathrm{s}}}-\left(\Delta \boldsymbol{E}_{\mathrm{op}}-\Delta \boldsymbol{E}_{\mathrm{eq}}\right)
$$

很容易从式(10)中得到

$$
\begin{aligned}
& \boldsymbol{P}^{\prime}=\Delta \boldsymbol{P}_{\mathrm{op}}-\Delta \boldsymbol{P}_{\mathrm{eq}} \\
& \boldsymbol{E}_{\boldsymbol{P}}=\Delta \boldsymbol{E}_{\mathrm{op}}-\Delta \boldsymbol{E}_{\mathrm{eq}}
\end{aligned}
$$

再根据 $\Delta \boldsymbol{P}_{\mathrm{op}}=\chi_{\mathrm{op}} \Delta \boldsymbol{E}_{\mathrm{op}}, \Delta \boldsymbol{P}_{\mathrm{eq}}=\chi_{\mathrm{s}} \Delta \boldsymbol{E}_{\mathrm{eq}}, \boldsymbol{E}_{\mathrm{ex}}$ 最终可以写为

$$
\begin{aligned}
\boldsymbol{E}_{\mathrm{ex}} & =\frac{\chi_{\mathrm{op}} \Delta \boldsymbol{E}_{\mathrm{op}}-\chi_{\mathrm{s}} \Delta \boldsymbol{E}_{\mathrm{eq}}}{\chi_{\mathrm{s}}}-\left(\Delta \boldsymbol{E}_{\mathrm{op}}-\Delta \boldsymbol{E}_{\mathrm{eq}}\right) \\
& =\left(\frac{\chi_{\mathrm{op}}}{\chi_{\mathrm{s}}}-1\right) \Delta \boldsymbol{E}_{\mathrm{op}}
\end{aligned}
$$

式(13)中 $\chi_{\mathrm{op}}$ 和 $\chi_{\mathrm{s}}$ 分别为溶剂介质的光频极化率和 静态极化率.

对于平衡态, 静电场的能量为 ${ }^{[16]}$

$$
G=\frac{1}{8 \pi} \int_{V} \boldsymbol{E} \cdot \boldsymbol{D} \mathrm{d} V=\frac{1}{8 \pi} \int_{V} \boldsymbol{E}_{\mathrm{vac}}^{2} \mathrm{~d} V-\frac{1}{2} \int_{V} \boldsymbol{E}_{\mathrm{vac}} \cdot \boldsymbol{P} \mathrm{d} V
$$

式(14)积分遍及整个空间 $V$, 右边第 1 项是溶质真 空电场自由能, $\boldsymbol{E}_{\mathrm{vac}}$ 是溶质真空电场. 第 2 项是溶剂 介质在真空电场下的能量(即溶剂化自由能). 那么约 束平衡态 $\left[\boldsymbol{E}_{2}^{*}, \boldsymbol{D}_{2}^{*}\right]$ 的静电自由能 $G_{2}^{*}$ 可以写为

$$
\begin{aligned}
G_{2}^{*} & =\frac{1}{8 \pi} \int_{V}\left(\boldsymbol{E}_{\mathrm{vac}, 2}+\boldsymbol{E}_{\mathrm{ex}}\right)^{2} \mathrm{~d} V-\frac{1}{2} \int_{V}\left(\boldsymbol{E}_{\mathrm{vac}, 2}+\boldsymbol{E}_{\mathrm{ex}}\right) \cdot \boldsymbol{P}_{2}^{\text {non }} \mathrm{d} V \\
& =\frac{1}{8 \pi} \int_{V}\left(\boldsymbol{E}_{\mathrm{vac}, 2}+\boldsymbol{E}_{\mathrm{ex}}\right)^{2} \mathrm{~d} V-\frac{1}{2} \int_{V}\left(\boldsymbol{E}_{\mathrm{vac}, 2}+\boldsymbol{E}_{\mathrm{ex}}\right) .
\end{aligned}
$$




$$
\left(\boldsymbol{P}_{2}^{\mathrm{eq}}+\boldsymbol{P}^{\prime}\right) \mathrm{d} V
$$

式(15)中的 $\boldsymbol{E}_{\mathrm{vac}, 2}$ 是平衡态 2 的溶质真空电场, 右边 的第 2 项正是约束平衡态 $\left[\boldsymbol{E}_{2}^{*}, \boldsymbol{D}_{2}^{*}\right]$ 的溶剂化自由能, 记为 $\Delta F_{2}^{*}$

$$
\Delta F_{2}^{*}=-\frac{1}{2} \int_{V}\left(\boldsymbol{E}_{\mathrm{vac}, 2}+\boldsymbol{E}_{\mathrm{ex}}\right) \cdot\left(\boldsymbol{P}_{2}^{\mathrm{eq}}+\boldsymbol{P}^{\prime}\right) \mathrm{d} V
$$

根据式(4)和(16), 可以得到非平衡态 $2\left[\boldsymbol{E}_{2}^{\text {non }}, \boldsymbol{D}_{2}^{\text {non }}\right]$ 的 溶剂化自由能为 $\Delta F_{2}^{\text {non }}$

$$
\begin{aligned}
\Delta F_{2}^{\mathrm{non}}= & \Delta F_{2}^{*}+\int_{V} \boldsymbol{E}_{\mathrm{ex}} \cdot \boldsymbol{P}_{2}^{\mathrm{non}} \mathrm{d} V=\Delta F_{2}^{*}+\int_{V} \boldsymbol{E}_{\mathrm{ex}} \cdot\left(\boldsymbol{P}_{2}^{\mathrm{eq}}+\boldsymbol{P}^{\prime}\right) \mathrm{d} V \\
= & -\frac{1}{2} \int_{V} \boldsymbol{E}_{\mathrm{vac}, 2} \cdot \boldsymbol{P}_{2}^{\mathrm{eq}} \mathrm{d} V+\frac{1}{2} \int_{V} \boldsymbol{E}_{\mathrm{ex}} \cdot \boldsymbol{P}^{\prime} \mathrm{d} V- \\
& \frac{1}{2} \int_{V} \boldsymbol{E}_{\mathrm{vac}, 2} \cdot \boldsymbol{P}^{\prime} \mathrm{d} V+\frac{1}{2} \int_{V} \boldsymbol{E}_{\mathrm{ex}} \cdot \boldsymbol{P}_{2}^{\mathrm{eq}} \mathrm{d} V
\end{aligned}
$$

式(17)中最后两项相互抵消(附录), 所以有

$$
\Delta F_{2}^{\mathrm{non}}=-\frac{1}{2} \int_{V} \boldsymbol{E}_{\mathrm{vac}, 2} \cdot \boldsymbol{P}_{2}^{\mathrm{eq}} \mathrm{d} V+\frac{1}{2} \int_{V} \boldsymbol{E}_{\mathrm{ex}} \cdot \boldsymbol{P}^{\prime} \mathrm{d} V
$$

根据溶剂重组能的定义, 式(18)右边第 1 项是平衡 态 2 的溶剂化自由能 $\Delta F_{2}^{\mathrm{eq}}$, 第 2 项就是溶剂重组能 $\lambda_{\mathrm{o}}$, 即

$$
\lambda_{\mathrm{o}}=\Delta F_{2}^{\mathrm{non}}-\Delta F_{2}^{\mathrm{eq}}=\frac{1}{2} \int_{V} \boldsymbol{E}_{\mathrm{ex}} \cdot \boldsymbol{P}^{\prime} \mathrm{d} V
$$

将式(11)和(13)代人式(19), 得到

$$
\begin{aligned}
\lambda_{\mathrm{o}} & =\frac{1}{2} \frac{\chi_{\mathrm{op}}-\chi_{\mathrm{s}}}{\chi_{\mathrm{s}}} \int_{V} \Delta \boldsymbol{E}_{\mathrm{op}} \cdot\left(\Delta \boldsymbol{P}_{\mathrm{op}}-\Delta \boldsymbol{P}_{\mathrm{eq}}\right) \mathrm{d} V \\
& =\frac{1}{2} \frac{\varepsilon_{\mathrm{op}}-\varepsilon_{\mathrm{s}}}{\varepsilon_{\mathrm{s}}-1} \int_{V} \Delta \boldsymbol{E}_{\mathrm{op}} \cdot\left(\Delta \boldsymbol{P}_{\mathrm{op}}-\Delta \boldsymbol{P}_{\mathrm{eq}}\right) \mathrm{d} V \\
& =\frac{1}{2} \frac{\varepsilon_{\mathrm{s}}-\varepsilon_{\mathrm{op}}}{\varepsilon_{\mathrm{s}}-1} \int_{V} \Delta \boldsymbol{E}_{\mathrm{op}} \cdot\left(\Delta \boldsymbol{P}_{\mathrm{eq}}-\Delta \boldsymbol{P}_{\mathrm{op}}\right) \mathrm{d} V
\end{aligned}
$$

式(20)中的 $\varepsilon_{\mathrm{op}}$ 和 $\varepsilon_{\mathrm{s}}$ 分别为溶剂介质的光频介电常 数和静态介电常数. 它们与 $\chi_{\mathrm{op}}$ 和 $\chi_{\mathrm{s}}$ 的关系为, $\varepsilon_{\mathrm{op}}=$ $\chi_{\mathrm{op}}+1, \varepsilon_{\mathrm{s}}=\chi_{\mathrm{s}}+1$. 因为我们考虑的溶剂介质为均匀介 质, 所以满足

$$
\nabla \cdot \boldsymbol{P}=0, \boldsymbol{n} \cdot \boldsymbol{P}=\sigma
$$

式(21)中 $\boldsymbol{P}$ 为极化强度, $\boldsymbol{n}$ 为孔穴表面法线, $\sigma$ 是孔 穴表面极化面电荷密度. 再有 $\Delta \boldsymbol{E}_{\mathrm{op}}$ 和 $\Delta \Phi_{\mathrm{op}}$ 的关系 为

$$
\Delta \boldsymbol{E}_{\mathrm{op}}=-\nabla\left(\Delta \Phi_{\mathrm{op}}\right)
$$

式(22)中的 $\Delta \Phi_{\mathrm{op}}$ 是溶质真空电势的变化与溶剂介
质电子极化电势的变化之和. 将式(21)和(22)代人式 (20), 可得

$$
\begin{aligned}
\lambda_{0}= & \frac{1}{2} \frac{\varepsilon_{\mathrm{s}}-\varepsilon_{\mathrm{op}}}{\varepsilon_{\mathrm{s}}-1} \int_{V} \nabla\left(-\Delta \Phi_{\mathrm{op}}\right) \cdot\left(\Delta \boldsymbol{P}_{\mathrm{eq}}-\Delta \boldsymbol{P}_{\mathrm{op}}\right) \mathrm{d} V \\
= & \frac{1}{2} \frac{\varepsilon_{\mathrm{s}}-\varepsilon_{\mathrm{op}}}{\varepsilon_{\mathrm{s}}-1} \int_{V} \nabla \cdot\left[-\Delta \Phi_{\mathrm{op}}\left(\Delta \boldsymbol{P}_{\mathrm{eq}}-\Delta \boldsymbol{P}_{\mathrm{op}}\right)\right]- \\
& \left(-\Delta \Phi_{\mathrm{op}}\right) \nabla \cdot\left(\Delta \boldsymbol{P}_{\mathrm{eq}}-\Delta \boldsymbol{P}_{\mathrm{op}}\right) \mathrm{d} V \\
= & \frac{1}{2} \frac{\varepsilon_{\mathrm{s}}-\varepsilon_{\mathrm{op}}}{\varepsilon_{\mathrm{s}}-1} \oint_{S}-\Delta \Phi_{\mathrm{op}}\left(\Delta \boldsymbol{P}_{\mathrm{eq}}-\Delta \boldsymbol{P}_{\mathrm{op}}\right) \cdot \mathrm{d} S \\
= & \frac{1}{2} \frac{\varepsilon_{\mathrm{s}}-\varepsilon_{\mathrm{op}}}{\varepsilon_{\mathrm{s}}-1} \oint_{S} \Delta \Phi_{\mathrm{op}}\left(\Delta \sigma_{\mathrm{op}}-\Delta \sigma_{\mathrm{eq}}\right) \mathrm{d} S
\end{aligned}
$$

式(23)中, 面积分在溶质孔穴表面 $S$ 进行, $\Delta \sigma_{\mathrm{op}}$ 是由 溶质真空场的变化 $\Delta \boldsymbol{E}_{\mathrm{vac}}=\boldsymbol{E}_{\mathrm{vac}, 2}-\boldsymbol{E}_{\mathrm{vac}, 1}$ 在 $\varepsilon_{\mathrm{op}}$ 的介质中 引起的孔穴表面极化电荷变化. $\Delta \sigma_{\mathrm{eq}}$ 是由 $\Delta \boldsymbol{E}_{\mathrm{vac}}$ 在 $\varepsilon_{\mathrm{s}}$ 的介质中引起的孔穴表面极化电荷变化. 式(23)即 溶剂重组能的普适公式.

\section{2 溶剂-导体界面的电子转移溶剂重组能}

对于溶剂-导体界面电子转移, 我们可以用球界面模型做简化近似处理. 如图 1(a)所示, 界面上方 溶剂介质的静态介电常数和光频介电常数分别为 $\varepsilon_{\mathrm{s}}$ 和 $\varepsilon_{\mathrm{op}}$, 界面下方为导体. 给体球孔穴 B 处于溶剂介 质中, 半径为 $r_{\mathrm{B}}$, 球心带有电荷 $q_{\mathrm{B}}$, 球心到界面距离 为 $d\left(d>r_{\mathrm{B}}\right)$. 界面以下的导体为受体. 电子转移反应可 看成是发生在球心和导体之间. 我们用镜像电荷法, 得到图 1(b)的图像, 球 $\mathrm{B}^{\prime}$ 是球 $\mathrm{B}$ 的像, 其球心的电 荷量 $q_{\mathrm{B}}^{\prime}$ 为

$$
q_{\mathrm{B}}^{\prime}=-q_{\mathrm{B}}
$$

球-界面模型的溶剂重组能 $\lambda_{\mathrm{o}}$ 是由给体球 $\mathrm{B}$ 表 面和界面两部分的溶剂重组能之和, 分别用 $\lambda_{\mathrm{B}}$ 和 $\lambda_{\text {int }}$ 表示. 由于在导体表面 $\Delta \Phi_{\mathrm{op}}=0$, 根据式(23), 有 $\lambda_{\text {int }}=0$. 在给体球 $\mathrm{B}$ 表面

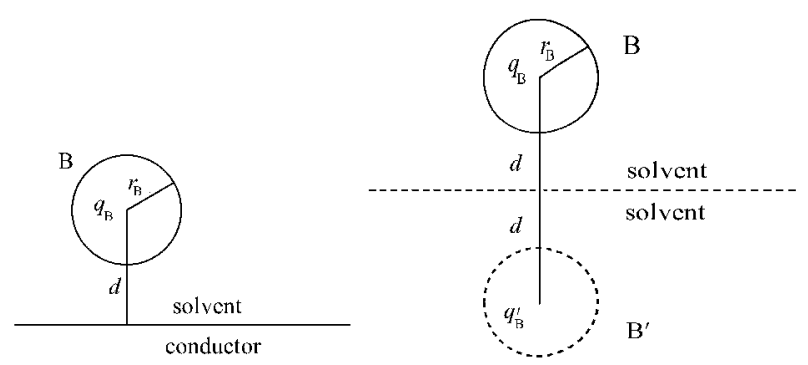

图 1 球-界面模型(a)和镜象电荷(b)示意图

Fig.1 Schematic descriptions of sphere-interface model (a) and image charge (b) 


$$
\begin{aligned}
\Delta \Phi_{\mathrm{op}} \approx \frac{\Delta q_{\mathrm{B}}^{\prime}}{2 \varepsilon_{\mathrm{op}} d} & +\frac{\Delta q_{\mathrm{B}}}{\varepsilon_{\mathrm{op}} r_{\mathrm{B}}}=\frac{\Delta q}{\varepsilon_{\mathrm{op}}}\left(\frac{1}{r_{\mathrm{B}}}-\frac{1}{2 d}\right) \\
\oint_{\mathrm{S}} \Delta \sigma_{\mathrm{op}}-\Delta \sigma_{\mathrm{eq}} \mathrm{d} S & =\frac{1-\varepsilon_{\mathrm{op}}}{\varepsilon_{\mathrm{op}}} \Delta q-\frac{1-\varepsilon_{\mathrm{s}}}{\varepsilon_{\mathrm{s}}} \Delta q \\
& =\left(\frac{1}{\varepsilon_{\mathrm{op}}}-\frac{1}{\varepsilon_{\mathrm{s}}}\right) \Delta q
\end{aligned}
$$

把式(25)、(26)代人式(23)得到

$$
\begin{aligned}
\lambda_{\mathrm{o}} & =\lambda_{\mathrm{B}} \approx \frac{1}{2} \frac{\varepsilon_{\mathrm{s}}-\varepsilon_{\mathrm{op}}}{\varepsilon_{\mathrm{s}}-1} \frac{\Delta q}{\varepsilon_{\mathrm{op}}}\left(\frac{1}{r_{\mathrm{B}}}-\frac{1}{2 d}\right)\left(\frac{1}{\varepsilon_{\mathrm{op}}}-\frac{1}{\varepsilon_{\mathrm{s}}}\right) \Delta q \\
& =\frac{(\Delta q)^{2}}{2} \frac{\varepsilon_{\mathrm{s}}-\varepsilon_{\mathrm{op}}}{\left(\varepsilon_{\mathrm{s}}-1\right) \varepsilon_{\mathrm{op}}}\left(\frac{1}{r_{\mathrm{B}}}-\frac{1}{2 d}\right)\left(\frac{1}{\varepsilon_{\mathrm{op}}}-\frac{1}{\varepsilon_{\mathrm{s}}}\right)
\end{aligned}
$$

与 Marcus 的结果 ${ }^{[22]}$

$$
\lambda_{\mathrm{o}, \text { Marcus }}=\frac{(\Delta q)^{2}}{2}\left(\frac{1}{r_{\mathrm{B}}}-\frac{1}{2 d}\right)\left(\frac{1}{\varepsilon_{\mathrm{op}}}-\frac{1}{\varepsilon_{\mathrm{s}}}\right)
$$

相比, 相差因子 $\frac{\varepsilon_{\mathrm{s}}-\varepsilon_{\mathrm{op}}}{\left(\varepsilon_{\mathrm{s}}-1\right) \varepsilon_{\mathrm{op}}}$. 对于极性溶剂, 式(27)的 计算结果约为式(28)的一半.

\section{2 算例结果与讨论}

用 C343(Coumarin 343)- $\mathrm{TiO}_{2}$ 体系(图 2)作为算 例来验证式(27). C343 分子的两个 $\mathrm{O}$ 原子分别通过 共价键和配位键与界面上的 $\mathrm{Ti}$ 原子相连. 文献[23] 测量并拟合了 $\mathrm{C} 343-\mathrm{TiO}_{2}$ 体系异相电子转移反应的 溶剂重组能, 并用式(28)验证了实验结果.

但是, 我们发现文献[23]所给的C343分子半径 值 $0.75 \mathrm{~nm}$ 太大, 大于C 343 分子重心到界面的距离 $0.5 \mathrm{~nm}$. 也就是说, C343 分子的球孔穴已经嵌人界面 以下, 这时式(27)和(28)均是不适用的. 于是, 我们用 Gaussian 03 $3^{[24]}$ 程序的标准方法估算了C343的分子体 积, 给出的推荐半径为 $0.458 \mathrm{~nm}$, 把 $r_{\mathrm{B}}=0.458 \mathrm{~nm} 、 d=$ $0.5 \mathrm{~nm} 、 \varepsilon_{\mathrm{s}}=24.3$ 和 $\varepsilon_{\mathrm{op}}=1.8526^{[23]}$ 分别代人式(27)和(28) 中, 得到 $\lambda_{0}=0.22 \mathrm{eV}$ 和 $\lambda_{\mathrm{o}, \mathrm{Marcus}}=0.42 \mathrm{eV}$. 很明显我们公 式的计算结果 $0.22 \mathrm{eV}$ 与实验值 $0.25 \mathrm{eV}^{[23]}$ 符合得更 好, 误差仅为 $12 \%$. 分析此误差主要来源于下面两 点: (1) $\mathrm{TiO}_{2}$ 不是完美导体; (2) 用 $r_{\mathrm{B}}=0.458 \mathrm{~nm}$ 的球

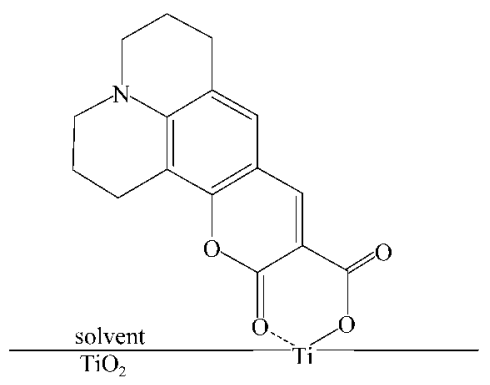

图 2 C343-TiO 2 体系的示意图

Fig.2 A depiction of C343-TiO $_{2}$ system
代替真实给体分子体系也存在误差. 但是, 无论从 定性还是定量上来看, 式(27)的计算结果是合理的.

\section{3 结 论}

非平衡态与其对应的平衡态之间的差别仅在于 溶剂介质取向极化的差异 $\boldsymbol{P}^{\prime}$. 借助 Leontovich 对非 平衡态的处理思想, 引人外加电场 $\boldsymbol{E}_{\mathrm{ex}}$ 对非平衡态 进行控制, 实现了把非平衡态控制成约束平衡态的 目标. 根据静电学基本原理和 Leontovich 提出的非 平衡态与控制平衡态能量关系式, 得到了正确普适 的非平衡溶剂化能和溶剂重组能的表达式. 基于球界面近似, 并结合镜象电荷法, 进一步推导得到了正 确的溶剂-导体界面电子转移溶剂重组能公式. 和 Marcus的公式相比, 我们的公式多了因子 $\left(\varepsilon_{\mathrm{s}}-\varepsilon_{\mathrm{op}}\right) /$ $\left(\varepsilon_{\mathrm{op}}\left(\varepsilon_{\mathrm{s}}-1\right)\right)$. 最后, 以 $\mathrm{C} 343-\mathrm{TiO}_{2}$ 体系为算例, 分别用 Marcus 和我们推导的公式计算了该体系溶剂重组 能, 并与实验值进行了比较. 结果表明, 我们的新理 论能给出更合理的计算结果.

致谢：本文理论工作得到北京大学刘文剑教授的帮助.

附录 式[17]中 $\int_{V} \boldsymbol{E}_{\mathrm{vac}, 2} \cdot \boldsymbol{P}^{\prime} \mathrm{d} V=\int_{V} \boldsymbol{E}_{\mathrm{ex}} \cdot \boldsymbol{P}_{2}^{\mathrm{eq}} \mathrm{d} V$ 的证明 根据平衡态时, $\boldsymbol{P}=\chi \mathbb{E}$, 有

$$
\int_{V} \boldsymbol{E}_{2}^{\mathrm{eq}} \cdot \boldsymbol{P}^{\prime} \mathrm{d} V=\int_{V} \boldsymbol{E}^{\prime} \cdot \boldsymbol{P}_{2}^{\mathrm{eq}} \mathrm{d} V
$$

这里 $\boldsymbol{E}^{\prime}=\boldsymbol{E}_{\mathrm{ex}}+\boldsymbol{E}_{\boldsymbol{P}}$. 再将电场 $\boldsymbol{E}_{2}^{\mathrm{eq}}$ 分为真空电场 $\boldsymbol{E}_{\mathrm{vac}, 2}$ 和极化电场 $\boldsymbol{E}_{\boldsymbol{P}, 2}$ 两部分, 式(Al)变成

$$
\begin{gathered}
\int_{V} \boldsymbol{E}_{\mathrm{vac}, 2} \cdot \boldsymbol{P}^{\prime} \mathrm{d} V+\int_{V} \boldsymbol{E}_{\boldsymbol{P}, 2} \cdot \boldsymbol{P}^{\prime} \mathrm{d} V=\int_{V} \boldsymbol{E}_{\mathrm{ex}} \cdot \boldsymbol{P}_{2}^{\mathrm{eq}} \mathrm{d} V+ \\
\int_{V} \boldsymbol{E}_{\boldsymbol{P}} \cdot \boldsymbol{P}_{2}^{\mathrm{eq}} \mathrm{d} V
\end{gathered}
$$

引人极化电势和极化电荷

$$
\begin{aligned}
\int_{V} \boldsymbol{E}_{\mathrm{P}, 2} \cdot \boldsymbol{P}^{\prime} \mathrm{d} V & =-\int_{S} \varphi_{2}^{\mathrm{eq}} \sigma^{\prime} \mathrm{d} S=-\int_{S} \int_{S^{\prime}} \frac{\sigma_{2}^{\text {eq }} \sigma^{\prime}}{\left|\boldsymbol{r}_{2}-\boldsymbol{r}^{\prime}\right|} \mathrm{d} S^{\prime} \mathrm{d} S \\
& =-\int_{S^{\prime}} \varphi^{\prime} \sigma_{2}^{\text {eq }} \mathrm{d} S^{\prime}=\int_{V} \boldsymbol{E}_{\boldsymbol{P}} \cdot \boldsymbol{P}_{2}^{\text {eq }} \mathrm{d} V
\end{aligned}
$$

这里 $\varphi_{2}^{\mathrm{eq}}$ 是平衡态 2 的极化电势, $\sigma^{\prime}$ 是 $\boldsymbol{P}^{\prime}$ 对应的极化面电 荷, $\sigma_{2}^{\mathrm{eq}}$ 是平衡态 2 时极化面电荷, $\left|\boldsymbol{r}_{2}-\boldsymbol{r}^{\prime}\right|$ 是极化面电荷 $\sigma_{2}^{\mathrm{eq}}$ 和 $\sigma^{\prime}$ 之间的距离. 将式 $\mathrm{A}(3)$ 代人式 $\mathrm{A}(2)$, 便得到

$$
\int_{V} \boldsymbol{E}_{\mathrm{vac}, 2} \cdot \boldsymbol{P}^{\prime} \mathrm{d} V=\int_{V} \boldsymbol{E}_{\mathrm{ex}} \cdot \boldsymbol{P}_{2}^{\mathrm{eq}} \mathrm{d} V
$$




\section{References}

1 Tomasi, J.; Mennucci, B.; Cammi, R. Chem. Rev., 2005, 105 : 2999

2 Marcus, R. A. J. Chem. Phys., 1956, 24: 979

3 Marcus, R. A. J. Chem. Phys., 1956, 24: 966

4 Marcus, R. A. J. Phys. Chem., 1994, 98: 7170

5 Miller, J. R.; Calcaterra, L. T.; Closs, G. L. J. Am. Chem. Soc., 1984, 106: 3047

6 Closs, G. L.; Calcaterra, L. T.; Green, N. J.; Penfield, K. W.; Miller, J. R. J. Phys. Chem., 1986, 90: 3673

7 Johnson, M. D.; Miller, J. R.; Green, N. S.; Closs, G. L. J. Phys. Chem., 1989, 93: 1173

8 Paulson, B. P.; Miller, J. R.; Gan, W. X.; Closs, G. L. J. Am. Chem. Soc., 2005, 127: 4860

9 Basilevsky, M. V.; Chudinov, G. E.; Rostov, I. V.; Liu, Y. P.; Newton, M. D. J. Mol. Struct.-Theochem, 1996, 371: 191

10 Newton, M. D.; Basilevsky, M. V.; Rostov, I. V. Chem. Phys., 1998, 232: 201

11 Basilevsky, M. V.; Rostov, I. V.; Newton, M. D. Chem. Phys., 1998, 232: 189

12 Blackbourn, R. L.; Hupp, J. T. J. Phys. Chem., 1990, 94: 1788
13 Formosinho, S. J.; Arnaut, L. G.; Fausto, R. Prog. React. Kinet. Mech., 1998, 23: 1

14 Serpa, C.; Gomes, P. J. S.; Arnaut, L. G.; de Melo, J. S.; Formosinho, S. J. ChemPhysChem, 2006, 7: 2533

15 Hupp, J. T.; Dong, Y.; Blackbourn, R. L. J. Phys. Chem., 1993, 97: 3278

16 Jackson, J. D. Classical electrodynamics. 3rd ed. New York: John Wiley \& Sons Press, 1999: 165-169

17 Li, X. Y.; Fu, K. X. J. Comput. Chem., 2004, 25: 500

18 Li, X. Y.; Fu, K. X.; Zhu, Q.; Shan, M. H. J. Comput. Chem., 2004, 25: 835

19 Fu, K. X.; Li, X. Y.; Zhu, Q.; Gong, Z.; Lu, S. Z.; Bao, Z. M. J. Mol. Struct. -Theochem, 2005, 715: 157

20 Li, X. Y.; Fu, K. X. J. Theor. Comput. Chem., 2005, 4: 907

21 Leontovich, M. A. An introduction to thermodynamics. 2nd ed. Moscow: Gittl Publ Press, 1950: §29, §30

22 Marcus, R. A. J. Chem. Phys., 1965, 43: 679

23 Moser, J. E.; Grätzel, M. Chem. Phys., 1993, 176: 493

24 Frisch, M. J.; Trucks, G. W.; Schlegel, H. B.; et al. Gaussian 03, Revision B. 01. Pittsburgh, PA: Gaussian, Inc., 2003 